\title{
Hemoptysis due to a large endobronchial mass successful regression after the use of high flow nasal cannula
}

\author{
Paola Pierucci, ${ }^{1}$ Nicolino Ambrosino, ${ }^{2}$ Annalisa Carlucci, ${ }^{3}$ Salvatore Grasso, ${ }^{4}$ \\ Giovanna Elisiana Carpagnano, ${ }^{1}$ Onofrio Resta ${ }^{1}$ \\ ${ }^{1}$ Department of Cardio Thoracic Medicine, Respiratory and Sleep Medicine Unit, "Aldo Moro" University of Bari \\ Policlinico Hospital, Bari; ${ }^{2}$ Department of Respiratory Medicine, ICS Maugeri IRCCS, Institute of Montescano (PV); \\ ${ }^{3}$ Department of Medicine and Surgery, Insubria University, Varese; ${ }^{4}$ Department of Emergency and Organ \\ Transplantation, Anaesthesiology and Critical care Unit, “Aldo Moro” University of Bari, Italy
}

\begin{abstract}
In recent years, high flow nasal cannula (HFNC) has being increasingly used. Most studies showed positive results of HFNC use in patients with hypoxemic respiratory failure. This manuscript reports a patient who presented with signs and symptoms related
\end{abstract}

Correspondence: Paola Pierucci, Department of Respiratory and Sleep Medicine, Chini Pavillion level 4, Policlinico di Bari, Piazza Giulio Cesare12, 70124 Bari.

Tel. +39.080.5591111.

E-mail: paola.pierucci@policlinico.ba.it

Key words: High flow nasal cannula; hemoptysis; hypoxic respiratory failure; HFNC; bronchial mass.

Contributions: PP, AC, GEC, gave substantial contributions to the conception or design of the work, the acquisition, and analysis; SG NA OR gave substantial contributions to the interpretation of the work, and drafting the work, revising it critically for important intellectual content, final approval of the version to be published, and agreement to be accountable for all aspects of the work in ensuring that questions related to the accuracy or integrity of any part of the work are appropriately investigated and resolved.

Conflict of interest: The authors declare no conflict of interest.

Informed consent: Patients' informed consent was collected prior the publication of material.

Acknowledgments: The authors would like to acknowledge Dr. BB as caregiver of the patient who was responsible for follow up and information.

Received for publication: 16 April 2020.

Accepted for publication: 18 June 2020.

${ }^{\circ}$ Copyright: the Author(s), 2020

Licensee PAGEPress, Italy

Monaldi Archives for Chest Disease 2020; 90:1321

doi: 10.4081/monaldi.2020.1321

This article is distributed under the terms of the Creative Commons Attribution Noncommercial License (by-nc 4.0) which permits any noncommercial use, distribution, and reproduction in any medium, provided the original author(s) and source are credited. to the presence of a large mass obstructing the right main bronchus: tachypnea, dyspnea, hemoptysis and hypoxemic respiratory failure. High flow oxygen via nasal cannula was initiated with a rapid improvement of the respiratory rate, dyspnea, hemoptysis, and the oxygen saturation. Thus, allowing the patient to be promptly stabilized.

\section{Introduction}

Over the past few years, the use of high flow nasal cannula (HFNC) oxygen delivery system has increasingly showed its benefits in patients with hypoxaemic respiratory failure (HRF) versus conventional oxygen reducing dyspnea and increasing fraction of Oxygen delivery [1-5]. Conventional oxygen systems via Venturi masks, is not well tolerated, major reasons being: the mask nuisance, the not sufficiently heated/humidified oxygen flow delivered, and the reduction in inspiratory oxygen fraction (FiO2) when the patient flow overcomes the total flow delivered by the mask [6-10]. Many studies have also highlighted the successful use of HFNC during invasive endotracheal procedures such as flexible bronchoscopy and intubation in hypoxemic patients [11-13]. The use of HFNC during rigid bronchoscopy in one case reporting the removal of large endotracheal mass emphasized the rapid preprocedure patient's oxygenation and its maintenance during the entire procedure [13]. Lastly, HFNC has been largely used in do not intubate or do not resuscitate patients, and in cancer patients with high effectiveness and improved patients' tolerance [14-18]. We report a case presenting with hemoptysis and hypoxemic respiratory failure due to a large endotracheal mass that successfully resolved after HFNC use.

\section{Case report}

The patient was a 64 years old Caucasian female presented at the emergency department of the Policlinico in Bari for dyspnoea at rest and hemoptysis. She had a positive familiar history for cancer. At arrival, she was afebrile, blood pressure was 120/85 $\mathrm{mmHg}$, rhythmic at $95 \mathrm{bpm}$ with pulse oximetry (SpO2) 89\% in room air. She was oriented, moderately tachypneic and dyspneic. The arterial blood gas analysis showed arterial oxygen tension $\left(\mathrm{PaO}_{2}\right): 59 \mathrm{mmHg}$ on air with normocapnia, therefore oxygen at $21 \mathrm{t} / \mathrm{min}$ was administered with prompt improvement in the pulse 
oximetry to $97 \%$. Bloods revealed $\mathrm{Hb} 13.8 \mathrm{~g} / \mathrm{L}$, PCR $21.2 \mathrm{mg} / \mathrm{dl}$, normal liver and renal function, no elevated D-Dimer or other flogosis markers. Laringoscopy excluded pharyngeal bleeding. The thoracic angio-CT-scan showed a voluminous mass extending from the last third of the trachea, partially invading the right main bronchus, mediastinal enlarged lymph-nodes, no source of bleeding or lung infiltrates/atelectasis were identified (Figure 1). Steroids, antibiotic and oxygen supplementation were initiated. Despite advices by caring physician and thoracic surgeon, she refused any surgical intervention. Over the next 24 hours, she continued to present further hemoptysis of increased amount with deterioration of dyspnea, hypoxaemia, requiring $\mathrm{FiO}_{2} 0.50$; ice packs were then applied over her chest. Repeated angio-CT chest highlighted a growing mass but still no arterial bleeding and no pulmonary emboli. Overnight of the second day, further deterioration occurred: dyspnea, accessory muscles recruitment, respiratory rate $35 \mathrm{bpm}$, stridor, hemoptysis (1-2 spoons of fresh blood, 3-4 times/hour, stable $\mathrm{Hb})$ and normocapnia but worsening hypoxaemia $\left(\mathrm{PaO}_{2}: 50\right.$ mmHg: $\mathrm{FiO}_{2}$ 0. 60 with $\mathrm{PaO}_{2} / \mathrm{FiO}_{2}$ ratio: 83). High Flow Nasal Cannula device was promptly available in our Unit and it was then initiated starting at the temperature of $34^{\circ} \mathrm{C}, 40 \mathrm{lt} / \mathrm{min}$ flow, and FiO2 0.60 (with AIRVO ${ }^{\text {TM }}$ Fisher \& Paykel). In 1 hour the patient's respiratory rate dropped to $18 \mathrm{bpm}$, the stridor ceased, the dyspnea improved. The patient presented one further hemoptysis episode of small amount overnight while the $\mathrm{PaO}_{2}$ increased to $68 \mathrm{mmHg}$ $\left(\mathrm{PaO}_{2} / \mathrm{FiO}_{2}: 115\right)$ with borderline normocapnia. The patient relaxed and fell asleep. During the following hours the situation continued to improve: only one further hemoptysis episode while the patient continued to use the HFNC, stable respiratory rate and $\mathrm{SpO}_{2}$ with reduced $\mathrm{FiO}_{2}$ requirements. However, despite medical advice, the patient refused to remain in hospital and she self-discharged home 5 days after the admission. It was suggested to continue taking tapering doses of steroids, to use at home the HFNC device, and to get urgent Thoracic-Surgery and Oncology consultation. Three weeks later a follow up call by her care provider reported that the patient was tapering doses of oral steroids but, conversely to what was suggested at the hospital, she was using conventional oxygen, and no surgical or oncological consult was performed. Unfortunately, one month after the admission she was found death overnight by her caregiver. No post mortem examination was performed.

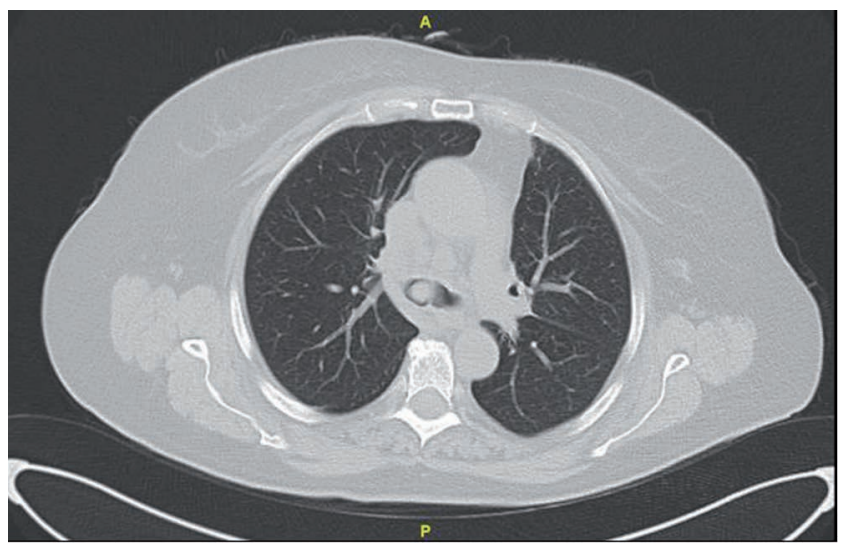

Figure 1. CT scan at trachea carina.

\section{Discussion and Conclusions}

High Flow Nasal Cannula (HFNC) has been described as very successful in the treatment of HRF of parenchymal source and in do not intubate or resuscitate orders [1-5, 14-16]. This is a case of tracheal mass partially occluding the main right main bronchus causing HRF and respiratory distress. As for the case reporting the successful and rapid pre-oxygenation and maintenance during excision of tracheal mass during rigid bronchoscopy [13], similarly, our patient promptly responded after HFNC application, successfully reducing her respiratory rate, improving dyspnea, hemoptysis and hypoxemia. We hypothesize several mechanisms that may have contributed to this rapid improvement. Firstly, warm and humidified air may have stabilized the desiccated and damaged bronchial mucosa optimizing its function, thus reducing cough, mucosal bleeding, improving gas exchange, and enhancing the secretions clearing [19]. Secondly, it may have helped reverting the bronchocostriction effect of cold, dry air which might have been of particular benefit in the partially obstructed airways, with further aid to the clearance of secretions and clots adhering to the tracheal lesions [20]. Thirdly, the generation of a small positive end expiratory pressure (PEEP) may have counteracted a possible initial alveolar de-recruitment due to the partial occlusion of the right main bronchus [21]. Lastly, HFNC may have reduced the work of breathing by increasingly reducing the driving pressure [22]. Further studies would be needed to confirm these results and elucidate mechanisms in similar patients' populations.

\section{References}

1. Pisani L, Astuto M, Prediletto I, Longhini F. High flow through nasal cannula in exacerbated COPD patients: a systematic review. Pulmonology 2019;25:348-54.

2. Nishimura M. High-flow nasal cannula oxygen therapy in adults: physiological benefits, indication, clinical benefits, and adverse effects. Respir Care 2016;61:529-41.

3. Vargas F, Saint-Leger M, Boyer A, et al. Physiologic effects of high-flow nasal cannula oxygen in critical care subjects. Respir Care 2015;60:1369-76.

4. Maggiore SM, Idone FA, Vaschetto R, et al. Nasal high-flow versus Venturi mask oxygen therapy after extubation. Effects on oxygenation, comfort, and clinical outcome. Am J Respir Crit Care Med 2014;190:282-8.

5. Frat J-P, Thille AW, Mercat A, et al. High-flow oxygen through nasal cannula in acute hypoxemic respiratory failure. $\mathrm{N}$ Eng $\mathrm{J}$ Med 2015;372:2185-96.

6. Lee CC, Mankodi D, Shaharyar S, et al. High flow nasal cannula versus conventional oxygen therapy and non-invasive ventilation in adults with acute hypoxemic respiratory failure: A systematic review. Respir Med 2016;121:100-8.

7. Brainard J, Scott BK, Sullivan BL, et al. Heated humidified high-flow nasal cannula oxygen after thoracic surgery - A randomized prospective clinical pilot trial. J Crit Care 2017;40:225-8.

8. Spoletini G, Alotaibi M, Blasi F, Hill NS. Heated humidified high-flow nasal oxygen in adults: mechanisms of action and clinical implications. Chest 2015;148:253-61.

9. Roca O, Hernandez G, Diaz-Lobato S, et al. Current evidence for the effectiveness of heated and humidified high flow nasal 
cannula supportive therapy in adult patients with respiratory failure. Crit Care 2016;20:109.

10. Chanques G, Constantin JM, Sauter M, et al. Discomfort associated with underhumidified high-flow oxygen therapy in critically ill patients. Intensive Care Med 2009;35:996-1003.

11. Lucangelo U, Vassallo FG, Marras E, et al. High-flow nasal interface improves oxygenation in patients undergoing bronchoscopy. Crit Care Res Pract 2012;2012:506382.

12. Miguel-Montanes R, Hajage D, Messika J, et al. Use of highflow nasal cannula oxygen therapy to prevent desaturation during tracheal intubation of intensive care patients with mildto-moderate hypoxemia. Crit Care Med 2015;43:574-83.

13. Min JY, Jo H, Roh K, Chung MY. Preventing deoxygenation with high flow nasal cannula oxygen during induction of general anesthesia for rigid bronchoscopy: Two case reports. Medicine (Baltimore) 2019;98:e15998.

14. Peters SG, Holets SR, Gay PC. High-flow nasal cannula therapy in do-not-intubate patients with hypoxemic respiratory distress. Respir Care 2013;58:597-600.

15. Epstein AS, Hartridge-Lambert SK, et al. Humidified high-flow nasal oxygen utilization in patients with cancer at Memorial Sloan-Kettering Cancer Center. J Palliat Med 2011;14:835-9.

16. Koyauchi T, Hasegawa H, Kanata K, et al. Efficacy and tolerability of high-flow nasal cannula oxygen therapy for hypoxemic respiratory failure in patients with interstitial lung disease with do-not-intubate orders: A retrospective singlecenter study. Respiration 2018;96:323-9.

17. Pierucci P, Carlucci C. End-stage respiratory diseases and respiratory support. Chapter 15 . In: C. Bausewein, DC Currow, MJ Johnson, editors. Palliative care in respiratory disease. ERS Monograph. European Respiratory Society; 2016.

18. Carlucci A, Vitacca M, Malovini A, et al. End-of-life discussion, patient understanding and determinants of preferences in very severe COPD patients: A multicentric study. COPD 2016;13:632-8

19. Williams R, Rankin N, Smith T, et al. Relationship between the humidity and temperature of inspired gas and the function of the airway mucosa. Crit Care Med 1996;24:1920-9.

20. Chidekel A, Zhu Y, Wang J, et al. The effects of gas humidification with high-flow nasal cannula on cultured human airway epithelial cells. Pulmonary Med 2012;2012:380686.

21. Corley A, Caruana LR, Barnett AG, et al. Oxygen delivery through high-flow nasal cannulae increase end-expiratory lung volume and reduce respiratory rate in post-cardiac surgical patients. Br J Anaesth 2011;107:998-1004.

22. Mundel T, Feng S, Tatkov S, Schneider H. Mechanisms of nasal high flow on ventilation during wakefulness and sleep. J Appl Physiol (1985) 2013;114:1058-65. 